Check for updates

Cite this: RSC Adv., 2020, 10, 18107

\title{
Development of TsDPEN based imine-containing ligands for the copper-catalysed asymmetric Kinugasa reaction $\uparrow$
}

\author{
Chuanlong Xu, \$ Yuchen Yang, \$Y Yue Wu, Feilong He, (D) Huakang He, Ping Deng* \\ and Hui Zhou (D)*
}

A novel class of chiral $N, N, N$ imine-containing ligands derived from TSDPEN ( $N$-( $p$-tosyl)-1,2diphenylethylene-1,2-diamine) has been developed and applied to the copper-catalyzed asymmetric Kinugasa reaction. The copper(II) salt proved to be an efficient catalyst precursor, and it provides an efficient way to synthesize enantioenriched cis- $\beta$-lactam. The pathway is air-tolerant and easily manipulated, and the ligands are easy to synthesize. A working model is proposed in which the stereocontrolling step is the [2+2] cycloaddition between ketene and imine to explain the observed stereoselectivities.

Received 12th April 2020

DOI: $10.1039 / \mathrm{dOra03276j}$

rsc.li/rsc-advances

reaction, such as phosphaferrocene-oxazoline, ${ }^{8 b}$ HETPHOX, ${ }^{10}$ Inda-bis(oxazoline), ${ }^{11}$ diamine, ${ }^{12} \mathrm{~N}$-PINAP, ${ }^{13}$ and Prolinol-Phos-

\section{Introduction}

Since the discovery of penicillin in 1928 , a number of $\beta$-lactam antibiotics have been developed. ${ }^{1}$ In addition to antibacterial activity, $\beta$-lactam containing molecules also exhibit special biological activities, ${ }^{2}$ such as Ezetimibe,${ }^{2 a}$ dual PPAR $\alpha / \gamma$ agonists, $^{2 b}$ a TRPV1 antagonist, ${ }^{2 c} \beta$-lactamic hFAAH inhibitors ${ }^{2 d}$ and neuronal protective effect against brain ischemia. ${ }^{2 e}$ At the same time, $\beta$-lactam compounds are also widely employed as useful intermediates in organic synthesis. ${ }^{3}$ Therefore, considerable efforts have been made in developing an efficient synthesis method for the preparation of chiral $\beta$-lactams. ${ }^{4}$

The Kinugasa reaction, developed in $1972,{ }^{5}$ is a simple and direct reaction between alkynes and nitrones for the synthesis of $\beta$-lactams in the presence of copper and a base. ${ }^{6}$ The group of Miura reported the first catalytic asymmetric version by using CuI (10 mol\%) and bis(oxazoline) (20 mol\%) with $57 \%$ ee and $35 \%$ de in $1995 .^{7} \mathrm{Fu}$ et al. achieved the first diastereoselective and enantioselective catalytic Kinugasa reaction by employing 1-2.5 mol\% CuCl and bis(azaferrocenes) (up to 93\% ee and cis/ trans up to 95/5) in 2002. ${ }^{8}$ Tang and co-workers demonstrated for the first time that a copper(II) catalyst could replace the previously performed air-sensitive copper(I) salts by using a $\mathrm{C}_{3^{-}}$symmetric tris(oxazoline) ligand in $2003 .{ }^{9}$ Since then, several ligands were developed for the catalytic asymmetric Kinugasa

Chongqing Research Center for Pharmaceutical Engineering, Research Center for Innovative Pharmaceutical and Excipient Analysis Technology, College of Pharmacy, Chongqing Medical University, Chongqing 400016, China. E-mail: hzhou@cqmu. edu.cn; orangedp@cqmu.edu.cn

$\dagger$ Electronic supplementary information (ESI) available. See DOI: 10.1039/d0ra03276j

\$ These authors contributed equally. phine. ${ }^{14}$ Enders et al. developed a method to synthesis spirocyclic $\beta$-lactams through Kinugasa/Michael domino reactions by the utilizing of copper-BOX..$^{15}$ In most cases the main products are $c i s-\beta$-lactams, except for the use of $\mathrm{Cu}$-diamine by Feng et al. ${ }^{12}$ Although progress have been achieved, the scope of chiral ligands employed in the Kinugasa reaction is limited and the catalytic highly enantioselective Kinugasa reaction remains a challenge and less developed.

Chiral imino-containing ligands have emerged as a powerful tool in asymmetric catalysis. ${ }^{16}$ Despite the great success that has been achieved, there is still a need to develop more powerful novel chiral ligands with imino group for asymmetric catalysis. ${ }^{16}$ Chan \& Li have designed two types of chiral tridentate $N$ -

(a) Previously reported chiral imine-containing ligands derived from TsDPEN

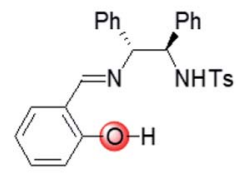

Chan \& Li, 2007

L1

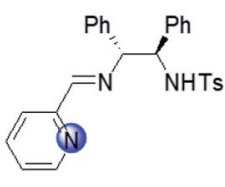

Li, 2009

L2 (b) Novel $N, N, N$ imine-containing ligands

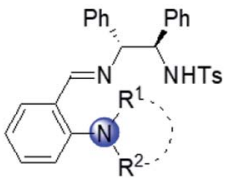

L3a: $R^{1}=R^{2}=M e$

L3b: $\mathrm{R}^{1}=\mathrm{R}^{2}=n \mathrm{Bu}$ L3c: $R^{1}, R^{2}=-\left(\mathrm{CH}_{2}\right)_{4}^{-}$ L3d: $\mathrm{R}^{1}, \mathrm{R}^{2}=-\left(\mathrm{CH}_{2}\right)_{5}$

Scheme 1 Chiral imine-containing ligands derived from TSDPEN. 
tosylated aminoimine ligands containing phenol and pyridine, respectively (Scheme 1a, $\mathbf{L 1}$ and $\mathbf{L} 2)^{17}$ for the addition of alkynes to imines. Recently, we have developed a new class of chiral polydentate $N, O$ ligands derived from $N$-( $p$-tosyl)-1,2diphenylethylene-1,2-diamine (TSDPEN). ${ }^{18,19}$ Herein, we successfully developed a novel class of chiral $N, N, N$ iminecontaining ligands derived from TsDPEN (Scheme 1b, L3) and applied them in $\mathrm{Cu}(\mathrm{II})$-catalysed asymmetric Kinugasa reaction.

\section{Results and discussion}

At the outset, we investigated the Kinugasa reaction of alkyne 1a and nitrone 2a in the presence of dicyclohexylamine as the base in $\mathrm{CH}_{3} \mathrm{CN}$ at $0{ }^{\circ} \mathrm{C}$, evaluating various copper catalysts prepared

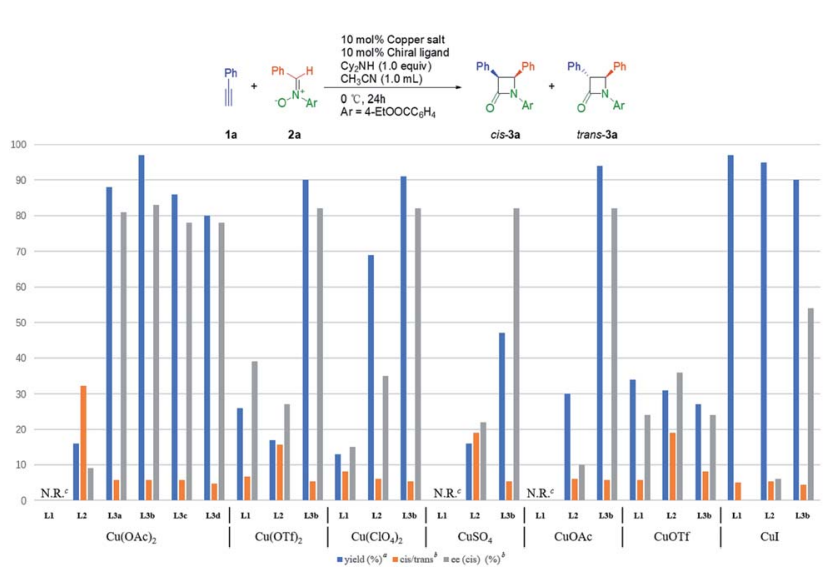

Fig. 1 Effect of copper salts and ligands on the asymmetric Kinugasa reaction. Reaction conditions: copper salt (10 mol\%), ligand (10 mol\%), 1a $(0.2 \mathrm{mmol}), 2 \mathrm{a}(0.22 \mathrm{mmol})$, dicyclohexylamine (1.0 equiv.), $\mathrm{CH}_{3} \mathrm{CN}$ $(1.0 \mathrm{~mL})$, at $0{ }^{\circ} \mathrm{C}$ for $24 \mathrm{~h}$. ${ }^{a}$ Total isolated yield of the cis- and transproducts. ${ }^{b}$ The ratios of cis/trans $(x / 1)$ and the ee of the cis-products were determined by HPLC on a chiral stationary phase. ${ }^{c} \mathrm{~N} . \mathrm{R} .=$ no reaction

Table 1 Effect of the base on the asymmetric Kinugasa reaction ${ }^{a}$

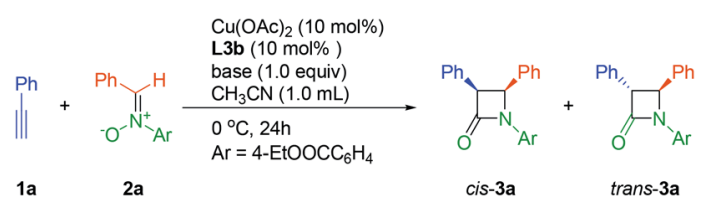

\begin{tabular}{lllll}
\hline Entry & Base & Yield $^{b}(\%)$ & cis/trans $^{c}$ & ee $(\text { cis })^{c}(\%)$ \\
\hline 1 & - & Trace & - & - \\
2 & $\mathrm{Cy}_{2} \mathrm{NH}^{d}$ & 97 & $85: 15$ & 83 \\
3 & $i \mathrm{Pr}_{2} \mathrm{NEt}$ & 10 & $87: 13$ & 44 \\
4 & $\mathrm{~K}_{2} \mathrm{CO}_{3}$ & 9 & $61: 39$ & 71 \\
5 & $i \mathrm{Pr}_{2} \mathrm{NH}$ & 73 & $80: 20$ & 79 \\
6 & $\mathrm{Cy}_{2} \mathrm{NMe}^{e}$ & 24 & $85: 15$ & 68 \\
7 & $n \mathrm{Bu}_{2} \mathrm{NH}$ & 90 & $59: 41$ & 80
\end{tabular}

${ }^{a}$ Reaction conditions: $\mathrm{Cu}(\mathrm{OAc})_{2}$ (10 mol\%), L3b (10 mol\%), 1 a $(0.2$ $\mathrm{mmol})$, $2 \mathrm{a}(0.22 \mathrm{mmol})$, base (1.0 equiv.), $\mathrm{CH}_{3} \mathrm{CN}(1.0 \mathrm{~mL})$, at $0{ }^{\circ} \mathrm{C}$ for $24 \mathrm{~h} .{ }^{b}$ Total isolated yield of the cis- and trans-products. ${ }^{c}$ Determined by HPLC on a chiral stationary phase. ${ }^{d}$ Dicyclohexylamine. ${ }^{e} N, N-$ Dicyclohexylmethylamine.
Table 2 Effect of the solvent on the Kinugasa reaction ${ }^{a}$

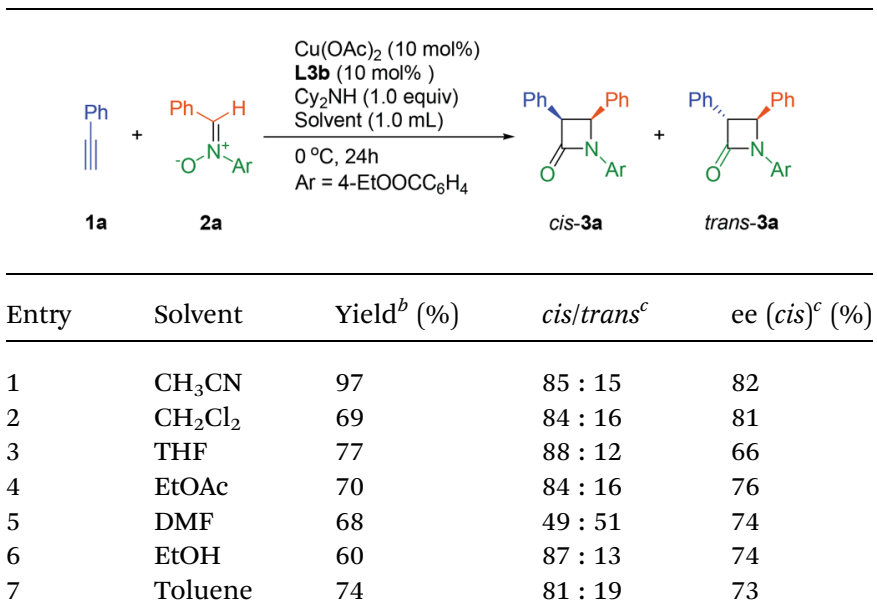

${ }^{a}$ Reaction conditions: $\mathrm{Cu}(\mathrm{OAc})_{2}$ (10 mol\%), L3b (10 mol\%), 1a $(0.2$ $\mathrm{mmol}), 2 \mathrm{a}(0.22 \mathrm{mmol})$, dicyclohexylamine (1.0 equiv.), solvent (1.0 $\mathrm{mL}$ ), at $0{ }^{\circ} \mathrm{C}$ for $24 \mathrm{~h} .{ }^{b}$ Total isolated yield of the cis- and transproducts. ${ }^{c}$ Determined by HPLC on a chiral stationary phase.

in situ from copper salts and chiral tridentate ligands L1-L3 (Fig. 1). ${ }^{20}$ Among them, $\mathrm{Cu}(\mathrm{OTf})_{2} / \mathbf{L 1}$ (26\% yield, $87 / 13$ cis/trans, $39 \%$ cis ee), ${ }^{21}$ CuOTf/L2 (31\% yield, 95/5 cis/trans, 36\% cis ee), ${ }^{22}$ $\mathrm{Cu}(\mathrm{OAc})_{2} / \mathbf{L} 3 \mathbf{b}$ (97\% yield, $85 / 15$ cis/trans, $83 \%$ cis ee) turned out to be the efficient combination in terms of yield, dr and enantioselectivity. These results indicated that the dialkylamine substitution in aniline obviously affected the reactivity and the enantioselectivity. Then the substituent effect on the amino group of $\mathbf{L} \mathbf{3}$ was examined and similar results were obtained ( $72-97 \%$ yield, $\sim 85 / 15$ cis/trans, $70-83 \%$ cis ee). Considering the reactivity and enantioselectivity, we selected $\mathbf{L} 3 \mathbf{b}$ for further optimization.

Next, the influences of different bases were studied with the results shown in Table 1. Although the ligand contained a tertiary amine functional group, this transformation hardly proceeded without basic additives (Table 1, entry 1). Tertiary amines and inorganic bases gave poor yield (Table 1, entries 3, 4 and 6). Secondary amines afforded the desired products with better yield and enantioselectivity, and higher sterically hindered secondary amines achieved higher diastereoselectivity (Table 1, entries 2, 5 and 7). Dicyclohexylamine proved to be the best base.

Solvent effects were studied next, with the results summarized in Table 2. Only moderate enantioselectivity could be obtained in examined solvents other than $\mathrm{CH}_{2} \mathrm{Cl}_{2}$ and $\mathrm{CH}_{3} \mathrm{CN}$ (Table 2, entries 1-7). No diastereoselectivity was observed in DMF (Table 2, entry 5). The best yield and enantioselectivity were obtained by $\mathrm{CH}_{3} \mathrm{CN}$ (Table 2, entry 1 ).

Subsequently, the reaction temperature was examined. As shown in Table 3, both the diastereoslectivity and enantioselectivity were dependent on the reaction temperature. When the temperature was decreased from 0 to $-30{ }^{\circ} \mathrm{C}$, the diastereoslectivity and enantioselectivity increased (Table 3, entries 13). When the reaction temperature further decreased to $-40{ }^{\circ} \mathrm{C}$, the reaction almost stopped by about halfway through (Table 3 , entry 4). 
Table 3 Effect of temperature on the asymmetric Kinugasa reaction ${ }^{a}$

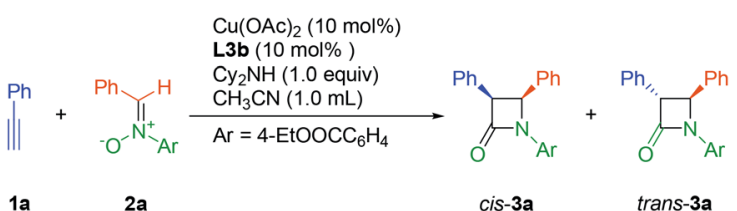

\begin{tabular}{llllll} 
Entry & $T\left({ }^{\circ} \mathrm{C}\right)$ & Time $(\mathrm{h})$ & Yield $^{b}(\%)$ & cis/trans & ee \\
\hline 1 & 0 & 24 & 97 & $85: 15^{c}$ & 82 \\
2 & -15 & 48 & $>99$ & $90: 10^{c}$ & 87 \\
3 & -30 & 48 & $>99$ & $91: 9^{c}(10.5: 1)^{d}$ & 90 \\
4 & -40 & 48 or 72 & 46 & $92: 8^{c}$ & 90
\end{tabular}

${ }^{a}$ Reaction conditions: $\mathrm{Cu}(\mathrm{OAc})_{2}(10 \mathrm{~mol} \%)$, L3b $(10 \mathrm{~mol} \%), 1 \mathrm{a}(0.2$ $\mathrm{mmol}), 2 \mathrm{a}(0.22 \mathrm{mmol})$, dicyclohexylamine (1.0 equiv.), $\mathrm{CH}_{3} \mathrm{CN}$ (1.0 $\mathrm{mL}) .{ }^{b}$ Total isolated yield of the cis- and trans-products. ${ }^{c}$ Determined by HPLC on a chiral stationary phase. ${ }^{d}$ Determined by ${ }^{1} \mathrm{H}$ NMR.

Under the established optimal reaction conditions, the substrate scope of the cycloaddition reaction between various alkynes and nitrones was examined next (Table 4). First, different alkynes participated in the reaction (Table 4, entries 1$6,12-15$, and 21). Weakly electron donating methyl group did not pose a problem for the reaction, and the corresponding cis$\beta$-lactams were isolated in good yields, diastereo- and

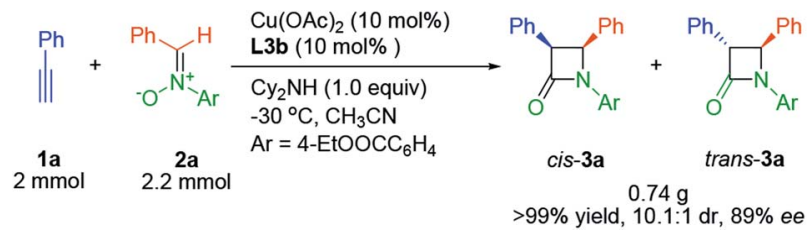

Scheme $2 \mathrm{mmol}$-scale reaction.

enantioselectivities (Table 4, entries 2, 3 and 12-15). The reaction conditions were also compatible with electron withdrawing group (Table 4, entries 4 and 21). Phenylacetylene with a strongly electron donating methoxy group had very low reactivity and only trace amount of corresponding product could be observed (Table 4, entry 5). The application of alkyl alkynes in the system is feasible, as exemplified by the formation of product 3e with high diastereoselectivity (99:1), albeit with low enantioselectivity (12\% ee). Next, effects of C-substituents of the nitrone were investigated (Table 4, entries 7-15). Methyl group and fused aromatic substituents had no adverse effect on the reaction. While strong decrease in reactivity for electron withdrawing group and heterocyclic substituents was observed (Table 4, entries 10 and 11). The electronic character of the $N$ bound aromatic groups of nitrones was also investigated (Table 4, entries 16-20). Whether electron-deficient or electron-rich $\mathrm{N}$ -

Table 4 Substrate scope of the asymmetric Kinugasa reaction ${ }^{a}$

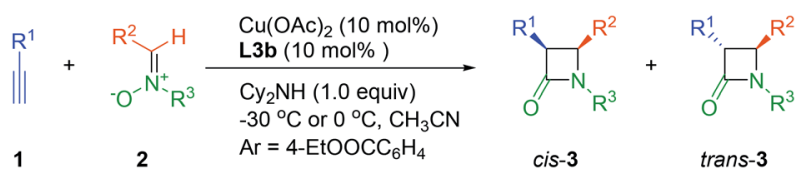

\begin{tabular}{|c|c|c|c|c|c|c|}
\hline Entry & $\mathrm{R}^{1}, \mathrm{R}^{2}, \mathrm{R}^{3}$ & $T\left({ }^{\circ} \mathrm{C}\right)$ & Product & cis/trans $^{b}$ & Yield $(c i s)^{c}(\%)$ & ee $(c i s)^{d}(\%)$ \\
\hline 1 & $\mathrm{C}_{6} \mathrm{H}_{5}, \mathrm{C}_{6} \mathrm{H}_{5}, \mathrm{Ar}$ & -30 & $3 \mathbf{a}$ & $10.5: 1$ & 92 & 90 \\
\hline 2 & $4-\mathrm{Me}-\mathrm{C}_{6} \mathrm{H}_{4}, \mathrm{C}_{6} \mathrm{H}_{5}, \mathrm{Ar}$ & -30 & $3 \mathbf{b}$ & $13.1: 1$ & 93 & 88 \\
\hline 3 & $3-\mathrm{Me}-\mathrm{C}_{6} \mathrm{H}_{4}, \mathrm{C}_{6} \mathrm{H}_{5}, \mathrm{Ar}$ & -30 & $3 c$ & $9.6: 1$ & 91 & 91 \\
\hline 4 & $4-\mathrm{F}-\mathrm{C}_{6} \mathrm{H}_{4}, \mathrm{C}_{6} \mathrm{H}_{5}, \mathrm{Ar}$ & 0 & $3 d$ & $6.2: 1$ & 74 & 88 \\
\hline 5 & $4-\mathrm{MeOC}_{6} \mathrm{H}_{4}, \mathrm{C}_{6} \mathrm{H}_{5}, \mathrm{Ar}$ & 0 & Trace & & & \\
\hline 6 & Cyclohexyl, $\mathrm{C}_{6} \mathrm{H}_{5}, \mathrm{Ar}$ & 0 & $3 e$ & $>99: 1$ & 68 & 12 \\
\hline 7 & $\mathrm{C}_{6} \mathrm{H}_{5}, 3-\mathrm{Me}-\mathrm{C}_{6} \mathrm{H}_{4}, \mathrm{Ar}$ & -30 & $3 f$ & $14: 1$ & 80 & 90 \\
\hline 8 & $\mathrm{C}_{6} \mathrm{H}_{5}, 4-\mathrm{Me}-\mathrm{C}_{6} \mathrm{H}_{4}, \mathrm{Ar}$ & 0 & $3 g$ & $6.7: 1$ & 81 & 83 \\
\hline 9 & $\mathrm{C}_{6} \mathrm{H}_{5}, 2$-naphthyl, Ar & 0 & $3 \mathbf{h}$ & $5.3: 1$ & 70 & 77 \\
\hline 10 & $\mathrm{C}_{6} \mathrm{H}_{5}, 4-\mathrm{Cl}-\mathrm{C}_{6} \mathrm{H}_{4}, \mathrm{Ar}$ & 0 & Trace & & & \\
\hline 11 & $\mathrm{C}_{6} \mathrm{H}_{5}$, 2-furyl, $\mathrm{Ar}$ & 0 & Trace & & & \\
\hline 12 & $4-\mathrm{Me}-\mathrm{C}_{6} \mathrm{H}_{4}, 3-\mathrm{Me}-\mathrm{C}_{6} \mathrm{H}_{4}, \mathrm{Ar}$ & -30 & $3 \mathbf{i}$ & $17.5: 1$ & 91 & 89 \\
\hline 13 & $3-\mathrm{Me}-\mathrm{C}_{6} \mathrm{H}_{4}, 3-\mathrm{Me}-\mathrm{C}_{6} \mathrm{H}_{4}, \mathrm{Ar}$ & -30 & $3 \mathbf{j}$ & $12.5: 1$ & 90 & 89 \\
\hline 14 & $4-\mathrm{Me}-\mathrm{C}_{6} \mathrm{H}_{4}, 4-\mathrm{Me}-\mathrm{C}_{6} \mathrm{H}_{4}, \mathrm{Ar}$ & 0 & $3 \mathbf{k}$ & $11: 1$ & 80 & 79 \\
\hline 15 & $3-\mathrm{Me}-\mathrm{C}_{6} \mathrm{H}_{4}, 4-\mathrm{Me}-\mathrm{C}_{6} \mathrm{H}_{4}, \mathrm{Ar}$ & 0 & 31 & $6.3: 1$ & 82 & 79 \\
\hline 16 & $\mathrm{C}_{6} \mathrm{H}_{5}, \mathrm{C}_{6} \mathrm{H}_{5}, \mathrm{C}_{6} \mathrm{H}_{5}$ & -30 & $3 \mathbf{m}$ & $12.9: 1$ & 93 & 91 \\
\hline 17 & $\mathrm{C}_{6} \mathrm{H}_{5}, \mathrm{C}_{6} \mathrm{H}_{5}, 4-\mathrm{Me}-\mathrm{C}_{6} \mathrm{H}_{4}$ & 0 & $3 n$ & $14: 1$ & 71 & 85 \\
\hline 18 & $\mathrm{C}_{6} \mathrm{H}_{5}, \mathrm{C}_{6} \mathrm{H}_{5}, 4-\mathrm{Cl}-\mathrm{C}_{6} \mathrm{H}_{4}$ & 0 & 30 & $12.5: 1$ & 80 & 87 \\
\hline 19 & $\mathrm{C}_{6} \mathrm{H}_{5}, \mathrm{C}_{6} \mathrm{H}_{5}, 4-\mathrm{Br}-\mathrm{C}_{6} \mathrm{H}_{4}$ & 0 & $3 \mathbf{p}$ & $11.8: 1$ & 82 & 87 \\
\hline 20 & $\mathrm{C}_{6} \mathrm{H}_{5}, \mathrm{C}_{6} \mathrm{H}_{5}, 4-\mathrm{F}-\mathrm{C}_{6} \mathrm{H}_{4}$ & 0 & $3 q$ & $16.5: 1$ & 70 & 86 \\
\hline 21 & $4-\mathrm{F}-\mathrm{C}_{6} \mathrm{H}_{4}, \mathrm{C}_{6} \mathrm{H}_{5}, \mathrm{C}_{6} \mathrm{H}_{5}$ & 0 & $3 \mathbf{r}$ & $16.3: 1$ & 75 & 84 \\
\hline
\end{tabular}

${ }^{a}$ Reaction conditions: $\mathrm{Cu}(\mathrm{OAc})_{2}(10 \mathrm{~mol} \%), \mathbf{L 3 b}(10 \mathrm{~mol} \%), \mathbf{1}(0.2 \mathrm{mmol}), 2(0.22 \mathrm{mmol})$, dicyclohexylamine $(1.0$ equiv. $), \mathrm{CH}_{3} \mathrm{CN}(1.0 \mathrm{~mL})$, at $-30{ }^{\circ} \mathrm{C}$ or $0{ }^{\circ} \mathrm{C}$ for $48 \mathrm{~h} .{ }^{b}$ Determined by ${ }^{1} \mathrm{H}$ NMR of the crude reaction mixture. ${ }^{c}$ Isolated yield of cis-product by chromatography on silica gel.

${ }^{d}$ Determined by HPLC on a chiral stationary phase. 


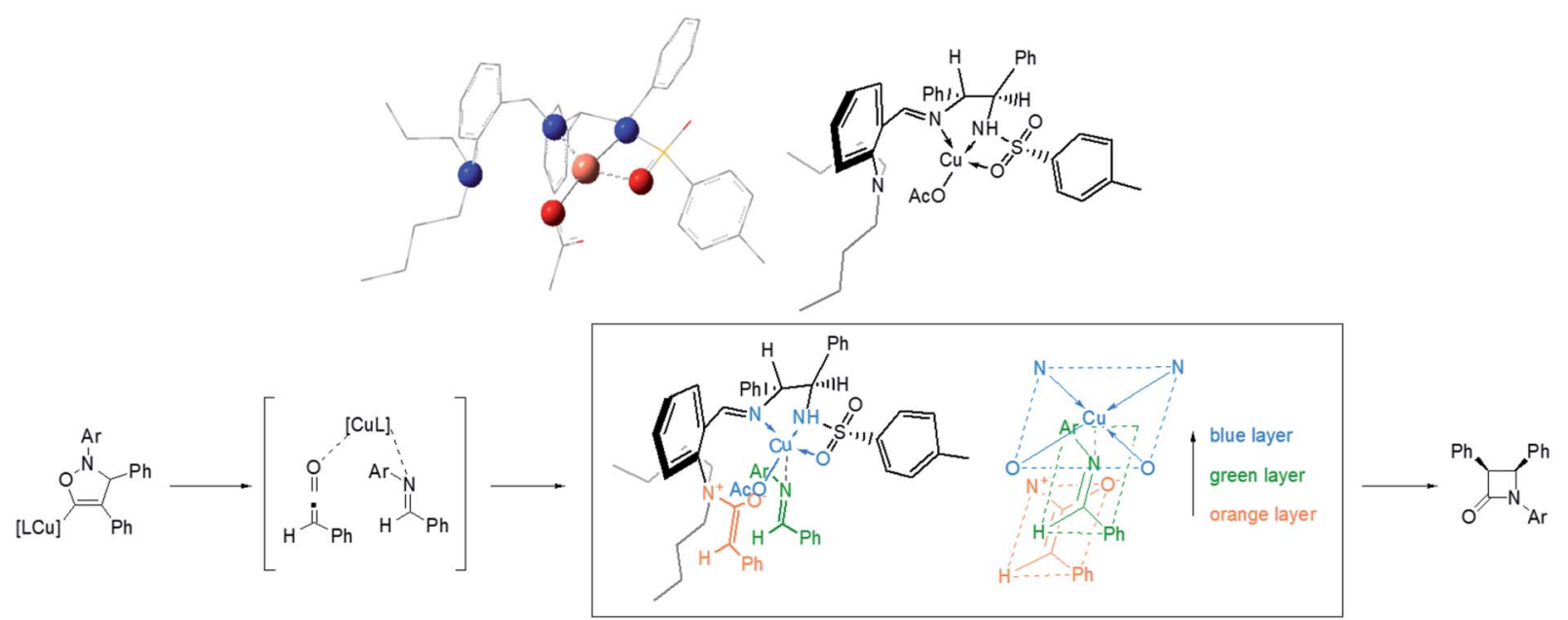

Fig. 2 Structure of the Cu complex optimized by DFT and proposed working model for stereoselectivity.

aryl nitrones were found to be highly suitable substrates for this asymmetric transformation.

To demonstrate the utility of this method, a mmol scale catalytic asymmetric Kinugasa reaction with $2.0 \mathrm{mmol} 1 \mathrm{a}$ and $2.2 \mathrm{mmol} 2 \mathrm{a}$ in the presence of $10 \mathrm{~mol} \%$ chiral copper complex proceeded smoothly and 3a was isolated with comparably high yield and stereoselectivity (Scheme 2).

The research of Kinugasa reaction mechanism has been an active research area. ${ }^{23}$ Recently, Hein's group reported an improved mechanism based on the analysis of multiple byproducts and detailed kinetic analysis. ${ }^{23 a}$ According to the modified mechanism, the stereocontrolling step is the $[2+2]$ cycloaddition between ketene and imine, which are generated by reductive elimination and protonation from the initial $[3+2]$ cycloaddition intermediate between nitrone and $\sigma$-Cu acetylide. Based on this research and the geometry of $\mathrm{Cu}$ complex optimized by DFT, ${ }^{24}$ a working model to explain the observed stereoselectivities is proposed in Fig. 2. The ketene combined with the tert-amine forming a zwitterionic enolate (phenyl away from $\mathrm{N}$ is favourable for the steric repulsion) and the imine coordinated to the $\mathrm{Cu}$ in a suitable position by avoiding the steric repulsion of $n$-butyl group on nitrogen. ${ }^{25}$

\section{Conclusions}

In summary, we have successfully employed the novel chiral $N, N, N$ imine-containing ligands derived from TsDPEN in the catalytic enantioselective Kinugasa reaction. It provides an efficient way to synthesize cis- $\beta$-lactam in good to excellent yields (up to 93\%) and with good to excellent diastereo- and enantioselectivities (dr up to 17.5 : 1, ee up to 91\%). This new protocol for the Kinugasa reaction is not effective for aliphatic alkynes and phenylacetylenes with a strongly electron donating group. A working model is proposed in which the stereocontrolling step is the $[2+2]$ cycloaddition between ketene and imine to explain the observed stereoselectivities. The $\mathrm{N}, \mathrm{N}$-dialkylamine substitution is an unprecedented modification on the TsDPEN based imine containing ligand scaffold. The pathway is air-tolerant and easily manipulated, and the ligands are easy to synthesize. Further investigations are underway in our laboratory including further geometry optimization of ligands, the detailed mechanism, and the extending of the substrate scope.

\section{Experimental section}

\section{General}

The solvents were dried by molecular sieve for more than $72 \mathrm{~h}$ before use. Other commercial reagents were used as purchased. NMR (Bruker $600 \mathrm{MHz}$ ) spectra were recorded in the deuterated solvents as stated, using residual non-deuterated solvent as internal standard. High resolution mass spectra were recorded with a Bruker Solari XFT-ICR-MS system. The enantiomeric excess (ee) was determined by HPLC (Shimadzu LC-16) analysis using the corresponding commercial chiral column as stated in the experimental procedures at $23{ }^{\circ} \mathrm{C}$ with $\mathrm{UV}$ detector $(254 \mathrm{~nm})$. Optical rotations were measured on a commercial polarimeter (Rudolph Autopol I) and are reported as follows: $[\alpha]_{\mathrm{D}}^{\mathrm{T}}(c=\mathrm{g} / 100$ $\mathrm{mL}$, solvent). The diastereomeric ratios of the $\beta$-lactams were determined by integration of ${ }^{1} \mathrm{H}$ NMR spectra (the coupling constant for $\mathrm{C} 3 / \mathrm{C} 4$ vicinal protons is $4.5-6 \mathrm{~Hz}$ for cis isomers and 2-2.5 Hz for trans isomers). ${ }^{8 a}$ The absolute configuration of $3 \mathbf{a}, 3 \mathbf{e}$ and $3 \mathbf{m}$ was determined by the comparison of the HPLC retention time and optical rotations with the literature data. ${ }^{\mathbf{8}, \mathbf{1 2}}$ The absolute configuration of $\mathbf{3 b - 3 d}, \mathbf{3} \mathbf{f}-\mathbf{3} \mathbf{l}$ and $\mathbf{3 n} \mathbf{n}-\mathbf{3 r}$ was determined by analogy.

\section{General preparation procedure of ligands}

L1 and L2 are known compounds. ${ }^{17}$

L1. Yellow solid, ${ }^{1} \mathrm{H}$ NMR (600 MHz, $\left.\mathrm{CDCl}_{3}\right) \delta 12.66(\mathrm{~s}, 1 \mathrm{H})$, $8.16(\mathrm{~s}, 1 \mathrm{H}), 7.41(\mathrm{~d}, J=8.0 \mathrm{~Hz}, 2 \mathrm{H}), 7.34-7.31(\mathrm{~m}, 1 \mathrm{H}), 7.19-7.07$ (m, 9H), 7.00-6.95 (m, 3H), 6.90-6.83 (m, 3H), $5.32(\mathrm{~d}, J=$ $7.4 \mathrm{~Hz}, 1 \mathrm{H}), 4.76(\mathrm{t}, J=6.7 \mathrm{~Hz}, 1 \mathrm{H}), 4.56(\mathrm{~d}, J=5.9 \mathrm{~Hz}, 1 \mathrm{H}), 2.30$ (s, 3H).

L2. Yellow solid, ${ }^{1} \mathrm{H}$ NMR $\left(600 \mathrm{MHz}, \mathrm{CDCl}_{3}\right) \delta 8.60(\mathrm{~d}, J=$ $4.5 \mathrm{~Hz}, 1 \mathrm{H}), 8.00$ (d, $J=7.7 \mathrm{~Hz}, 1 \mathrm{H}), 7.85-7.83$ (m, 1H), 7.62 (d, $J$ 
$=8.2 \mathrm{~Hz}, 2 \mathrm{H}), 7.32-7.30(\mathrm{~m}, 1 \mathrm{H}), 7.22-7.16(\mathrm{~m}, 10 \mathrm{H}), 6.99(\mathrm{~d}, J=$ $7.1 \mathrm{~Hz}, 1 \mathrm{H}), 5.94(\mathrm{~s}, 1 \mathrm{H}), 4.64(\mathrm{~d}, J=6.6 \mathrm{~Hz}, 1 \mathrm{H}), 4.32(\mathrm{~d}, J=$ $6.6 \mathrm{~Hz}, 1 \mathrm{H}), 2.43$ (s, 3H).

General preparation procedure of $\mathbf{L} 3$. To a solution of $o$-fluorobenzaldehyde $(10 \mathrm{mmol})$ and a secondary-amine $(20 \mathrm{mmol})$ in $20 \mathrm{~mL}$ DMF was added $\mathrm{K}_{2} \mathrm{CO}_{3}(20 \mathrm{mmol})$, and the mixture was stirred under a $\mathrm{N}_{2}$ atmosphere at $75{ }^{\circ} \mathrm{C}$ until the reaction proceed completely detected by TLC. The reaction mixture was filtered, the filtrate was diluted with $\mathrm{CH}_{2} \mathrm{Cl}_{2}(50 \mathrm{~mL})$ and washed with saturated sodium bicarbonate solution $(20 \mathrm{~mL} \times 3)$. The organic layers were dried $\left(\mathrm{Na}_{2} \mathrm{SO}_{4}\right)$, and purified through column chromatography (petroleum ether/ether, 10:1) on silica gel to obtain a crude product. The crude product was dissolved in the mixture of petroleum ether/ether $(v / v \quad 10: 1)$ and acidified to $\mathrm{pH} 2$ with $1 \mathrm{M} \mathrm{HCl}$. The resulting mixture was extracted twice with water, and the aqueous layer was basified to pH 9 with $1 \mathrm{M} \mathrm{NaOH}$ and extracted with $\mathrm{CH}_{2} \mathrm{Cl}_{2}$. The dichloromethane layers were dried and the solvents were removed in vacuo to afford 4 . To a solution of $4(1.2 \mathrm{mmol})$ in $30 \mathrm{~mL}$ ethanol was added $5(1.2 \mathrm{mmol})$ and the mixture was stirred at $75{ }^{\circ} \mathrm{C}$ under a $\mathrm{N}_{2}$ atmosphere until the reaction proceed completely detected by TLC. The EtOH was removed in vacuo to give $\mathbf{L} \mathbf{3}$.

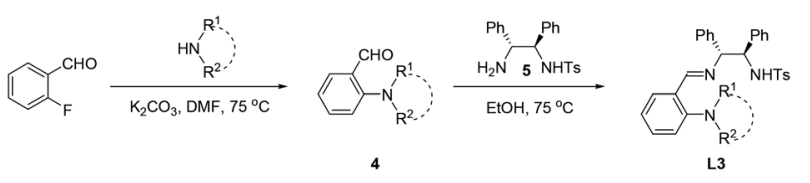

L3a. White solid, (mixture of $E$ - and $Z-, E / Z=5.6 / 1$ ), ${ }^{1} \mathrm{H}$ NMR $\left(600 \mathrm{MHz}, \mathrm{CDCl}_{3}\right) \delta 8.14(E, \mathrm{~s}, 1 \mathrm{H}), 7.97(E, \mathrm{~d}, J=7.4 \mathrm{~Hz}, 1 \mathrm{H})$, $7.91(Z, \mathrm{~d}, J=7.5 \mathrm{~Hz}, 0.18 \mathrm{H}), 7.56(Z, \mathrm{~d}, J=8.0 \mathrm{~Hz}, 0.37 \mathrm{H}), 7.51$ $(Z, \mathrm{~d}, J=7.5 \mathrm{~Hz}, 0.38 \mathrm{H}), 7.32-7.29(E+Z, \mathrm{~m}, 3.96 \mathrm{H}), 7.19-7.00(E$ $+Z, \mathrm{~m}, 11.37 \mathrm{H}), 6.91-6.89(E+Z, \mathrm{~m}, 3.12 \mathrm{H}), 6.19(E, \mathrm{~d}, J=$ $7.0 \mathrm{~Hz}, 1 \mathrm{H}) 4.84(E, \mathrm{q}, J=3.3 \mathrm{~Hz}, 1 \mathrm{H}), 4.77(Z, \mathrm{~d}, J=5.2 \mathrm{~Hz}$, $0.18 \mathrm{H}), 4.51(E, \mathrm{~d}, J=3.4 \mathrm{~Hz}, 1 \mathrm{H}), 4.26(Z, \mathrm{~d}, J=5.3 \mathrm{~Hz}, 0.18 \mathrm{H})$, $2.35(E, \mathrm{~s}, 6 \mathrm{H}), 2.32(Z, \mathrm{~s}, 0.71 \mathrm{H}), 2.25(E, \mathrm{~s}, 3 \mathrm{H}), 2.23(Z, \mathrm{~s}$, $0.20 \mathrm{H}) .{ }^{13} \mathrm{C} \mathrm{NMR}\left(150 \mathrm{MHz}, \mathrm{CDCl}_{3}\right) \delta E: 162.1,154.1,142.2$, 140.9, 140.0, 137.2, 131.5, 129.0, 121.9, 117.8, 78.5, 63.6, 45.0, 21.3; Z: 162.1, 152.5, 143.4, 140.02, 139.4, 135.5, 133.4, 129.2, 124.0, 120.2, 75.2, 71.6, 68.5, 45.4, 21.4.; $E+Z: 128.27,128.22$, $127.98,127.95,127.84,127.5,127.4,127.03,127.02,126.90$, 126.89, 126.7, 126.5. ESI-HRMS calcd for $\mathrm{C}_{30} \mathrm{H}_{32} \mathrm{~N}_{3} \mathrm{O}_{2} \mathrm{~S}^{+}[\mathrm{M}+$ $\mathrm{H}]^{+}:$498.2210, found 498.2200.

L3b. White solid, (mixture of $E$ - and $Z$-, $E / Z=9.1 / 1$ ), ${ }^{1} \mathrm{H}$ NMR $\left(600 \mathrm{MHz}, \mathrm{CDCl}_{3}\right) \delta 8.27(E, \mathrm{~s}, 1 \mathrm{H}), 8.03(E, \mathrm{~d}, J=7.3 \mathrm{~Hz}, 1 \mathrm{H})$, $7.65(Z, \mathrm{~d}, J=7.9 \mathrm{~Hz}, 0.23 \mathrm{H}), 7.42(Z, \mathrm{~d}, J=7.6 \mathrm{~Hz}, 0.25 \mathrm{H}), 7.34-$ $7.33(E+Z, \mathrm{~m}, 3 \mathrm{H}), 7.17-7.00(E+Z, \mathrm{~m}, 12 \mathrm{H}), 6.93(E, \mathrm{~d}, J=$ $7.9 \mathrm{~Hz}, 2 \mathrm{H}), 6.28,(Z, \mathrm{~s}, 0.11 \mathrm{H}), 6.20(E, \mathrm{~d}, J=6.5 \mathrm{~Hz}, 1 \mathrm{H}), 4.82(E$, $\mathrm{q}, J=3.5 \mathrm{~Hz}, 1 \mathrm{H}), 4.64(Z, \mathrm{~d}, J=6.4 \mathrm{~Hz}, 0.11 \mathrm{H}), 4.51(E, \mathrm{~d}, J=$ $3.7 \mathrm{~Hz}, 1 \mathrm{H}), 4.28(Z, \mathrm{~d}, J=6.4 \mathrm{~Hz}, 0.11 \mathrm{H}), 3.00-2.95(Z, \mathrm{~m}$, $0.49 \mathrm{H}), 2.73-2.64(E, \mathrm{~m}, 4 \mathrm{H}), 2.28$ ( $E, \mathrm{~s}, 3 \mathrm{H}), 2.27(Z, \mathrm{~s}, 0.16 \mathrm{H})$, 1.20-1.15 (E, m, 4H), 1.03-0.96 (E, m, 4H), $0.71(E, \mathrm{t}, J=7.4 \mathrm{~Hz}$, $6 \mathrm{H}) .{ }^{13} \mathrm{C} \mathrm{NMR}\left(150 \mathrm{MHz}, \mathrm{CDCl}_{3}\right) \delta E: 162.5,152.5,142.3,141.1$, 140.0, 137.4, 131.2, 78.0, 63.7, 54.2, 29.0, 21.4, 20.3, 13.9; Z: $162.5,150.0,143.5,139.6,139.2,137.3,130.7,75.8,71.9,68.5$, 54.6, 31.6, 29.4, 22.6, 21.5, 20.6, 20.3, 14.1, 14.0; $E+Z: 129.3$,
129.1, 128.3, 128.25, 128.20, 128.1, 128.0, 127.9, 127.7, 127.6, 127.3, 127.2, 127.08, 127.06, 126.9, 126.8, 124.2, 122.9, 122.8, 121.7. ESI-HRMS calcd for $\mathrm{C}_{36} \mathrm{H}_{44} \mathrm{~N}_{3} \mathrm{O}_{2} \mathrm{~S}^{+}[\mathrm{M}+\mathrm{H}]^{+}$: 582.3149, found 582.3140 .

L3c. White solid, (mixture of $E$ - and $Z-, E / Z=14.3 / 1$ ), ${ }^{1} \mathrm{H}$ NMR $\left(600 \mathrm{MHz}, \mathrm{CDCl}_{3}\right) \delta 8.10(E, \mathrm{~s}, 1 \mathrm{H}), 7.88(E, \mathrm{~d}, J=7.7 \mathrm{~Hz}, 1 \mathrm{H})$, $7.60(Z, \mathrm{~d}, J=7.8 \mathrm{~Hz}, 0.15 \mathrm{H}), 7.46(Z, \mathrm{~d}, J=7.5 \mathrm{~Hz}, 0.17 \mathrm{H}), 7.31-$ $7.25(E+Z, \mathrm{~m}, 3 \mathrm{H}), 7.23-7.09(E+Z, \mathrm{~m}, 10 \mathrm{H}), 6.9-6.8(E+Z, \mathrm{~m}$, $3.1 \mathrm{H}), 6.73(E+Z, \mathrm{~d}, J=8.3 \mathrm{~Hz}, 1 \mathrm{H}), 6.19(E+Z, \mathrm{~s}, 1.06 \mathrm{H}), 4.83$ $(E, \mathrm{q}, J=3.2 \mathrm{~Hz}, 1 \mathrm{H}), 4.69(Z, \mathrm{~d}, J=5.8 \mathrm{~Hz}, 0.07 \mathrm{H}), 4.47(E, \mathrm{~d}, J=$ $3.2 \mathrm{~Hz}, 1 \mathrm{H}), 4.32(Z, \mathrm{~d}, J=5.8 \mathrm{~Hz}, 0.07 \mathrm{H}), 2.80-2.76(E+Z, \mathrm{~m}$, $4 \mathrm{H}), 2.37(Z, \mathrm{~s}, 0.24 \mathrm{H}), 2.29(E, \mathrm{~s}, 3 \mathrm{H}), 1.69-1.64(E+Z, \mathrm{~m}$, 4.19H). ${ }^{13} \mathrm{C}$ NMR (150 MHz, $\left.\mathrm{CDCl}_{3}\right) \delta E: 163.2,150.4,142.3$, $141.0,140.2$, 137.3, 131.3, 129.2, 129.1, 128.3, 128.0, 127.06, 127.04, 126.98, 126.90, 126.7, 124.1, 118.9, 114.9, 78.7, 63.6, 52.6, 25.3, 21.4. ESI-HRMS calcd for $\mathrm{C}_{32} \mathrm{H}_{34} \mathrm{~N}_{3} \mathrm{O}_{2} \mathrm{~S}^{+}[\mathrm{M}+\mathrm{H}]^{+}$: 524.2366, found 524.2358.

L3d. White solid, (mixture of $E$ - and $Z-, E / Z=3.7 / 1$ ), ${ }^{1} \mathrm{H}$ NMR $\left(600 \mathrm{MHz}, \mathrm{CDCl}_{3}\right) \delta 8.13(E, \mathrm{~s}, 1 \mathrm{H}), 8.02(E, \mathrm{~d}, J=7.7 \mathrm{~Hz}, 1 \mathrm{H})$, $7.66(Z, \mathrm{~d}, J=7.6 \mathrm{~Hz}, 0.57 \mathrm{H}), 7.58(Z, \mathrm{~d}, J=7.8 \mathrm{~Hz}, 0.58 \mathrm{H}), 7.42$ $(E+Z, \mathrm{t}, J=7.6 \mathrm{~Hz}, 0.61 \mathrm{H}), 7.32-7.31(E+Z, \mathrm{~m}, 3.49 \mathrm{H}), 7.25-7.23$ $(E+Z, \mathrm{~m}, 1.87 \mathrm{H}), 7.18-7.05(E+Z, \mathrm{~m}, 12 \mathrm{H}), 6.92-6.90(E+Z, \mathrm{~m}$, $3.14 \mathrm{H}), 6.20-6.19(E+Z, \mathrm{~m}, 1.24 \mathrm{H}), 4.91(Z, \mathrm{~d}, J=3.4,0.27 \mathrm{H})$, $4.84-4.83(E, \mathrm{~m}, 1 \mathrm{H}), 4.53(Z, \mathrm{~s}, 0.27 \mathrm{H}), 4.48(E, \mathrm{~d}, J=3.5 \mathrm{~Hz}$, $1 \mathrm{H}), 2.59-2.57(E+Z, \mathrm{~m}, 2 \mathrm{H}), 2.49-2.46(E+Z, \mathrm{~m}, 2 \mathrm{H}), 2.29(Z, \mathrm{~s}$, $0.9 \mathrm{H}), 2.26(E, \mathrm{~s}, 3 \mathrm{H}), 1.37-1.30(E+Z, \mathrm{~m}, 6 \mathrm{H}) .{ }^{13} \mathrm{C}$ NMR (150 $\left.\mathrm{MHz}, \mathrm{CDCl}_{3}\right) \delta E: 162.0,154.5,142.2,140.9,140.0,131.6,78.5$, 63.6, 54.5, 26.0, 23.9, 21.3; Z: 162.0, 152.9, 143.3, 141.1, 139.8, 137.7, 135.6, 134.8, 133.2, 75.0, 70.7, 68.4, 26.7, 26.1, 24.3, 21.4; $E+Z: 129.1,129.02,128.98,128.95,128.5,128.45,128.43$, 128.29 , 128.26, 128.15, 128.07, 128.04, 127.95, 127.7, 127.5, 127.2, 127.1, 127.0, 127.95, 126.7, 126.6, 126.2, 125.2, 124.1, 122.3, 120.3, 118.6. ESI-HRMS calcd for $\mathrm{C}_{33} \mathrm{H}_{36} \mathrm{~N}_{3} \mathrm{O}_{2} \mathrm{~S}^{+}[\mathrm{M}+\mathrm{H}]^{+}$: 538.2523 , found 538.2518 .

\section{General procedure for the asymmetric Kinugasa reaction}

The mixture of $\mathrm{Cu}(\mathrm{OAc})_{2}(0.02 \mathrm{mmol}, 10 \mathrm{~mol} \%)$ and $\mathbf{L 3 b}$ (0.02 mmol, $10 \mathrm{~mol} \%$ ) was stirring in $\mathrm{CH}_{3} \mathrm{CN}(0.5 \mathrm{~mL})$ under air atmosphere at $35{ }^{\circ} \mathrm{C}$ for $1 \mathrm{~h}$. Then, dicyclohexylamine $(0.2$ $\mathrm{mmol})$ and alkyne $\mathbf{1}(0.2 \mathrm{mmol})$ were sequentially added, and the test tube was rinsed with $\mathrm{CH}_{3} \mathrm{CN}(0.2 \mathrm{~mL})$, after stirring at room temperature for 10 minutes, the mixture was cooled to $-30{ }^{\circ} \mathrm{C}$ or $0{ }^{\circ} \mathrm{C}$. After stirring for $10 \mathrm{~min}$ at $-30{ }^{\circ} \mathrm{C}$ or $0{ }^{\circ} \mathrm{C}$, nitrone $2(0.22 \mathrm{mmol})$ and $\mathrm{CH}_{3} \mathrm{CN}(0.3 \mathrm{~mL})$ were added. The mixture was further stirred at $-30{ }^{\circ} \mathrm{C}$ or $0{ }^{\circ} \mathrm{C}$ for the time indicated in Table 4 . The resulting solution was purified by column chromatography (petroleum ether/AcOEt) on silica gel to afford the products.

(3S,4S)-1-(4-Carboethoxyphenyl)-3,4-diphenyl-2-azetidinone (3a). White solid, $68.3 \mathrm{mg}, 92 \%$ yield (cis), 90\% ee. $[\alpha]_{\mathrm{D}}^{20}=-11.3$ (c $0.80, \mathrm{CHCl}_{3}$ ). The ee was determined by HPLC analysis using a CHIRALPAK IA column, hexane/2-propanol 90/10, flow rate $=$ $1.0 \mathrm{~mL} \mathrm{~min}{ }^{-1}, 254 \mathrm{~nm}$, cis: $t_{\mathrm{r}}=14.6 \mathrm{~min}(3 S 4 S$, major $)$ and $t_{\mathrm{r}}=$ $15.9 \min \left(3 R 4 R\right.$, minor); trans: $t_{\mathrm{r}}=17.5 \mathrm{~min}$ and $t_{\mathrm{r}}=18.3 \mathrm{~min}$ (ref. 12: CHIRALPAK IA column, hexane/2-propanol 90/10, flow

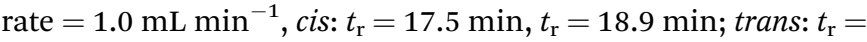


$\left.22.1 \min (3 S 4 R), t_{\mathrm{r}}=23.6 \mathrm{~min}(3 R 4 S)\right)$. The ee was also determined by HPLC analysis using a Chiralcel OD-H column, hexane/2-propanol 95/5, flow rate $=1.0 \mathrm{~mL} \mathrm{~min}^{-1}$, cis: $t_{\mathrm{r}}=$ $25.3 \mathrm{~min}\left(3 S 4 S\right.$, major) and $t_{\mathrm{r}}=35.8 \mathrm{~min}$ (3R4R, minor); trans: $t_{\mathrm{r}}$ $=12.8 \mathrm{~min}$ and $t_{\mathrm{r}}=16.3 \mathrm{~min}$ (ref. $8 a$ : Chiralcel OD, hexane/2propanol 95/5, flow rate $=1.0 \mathrm{~mL} \min ^{-1}$, cis: $t_{\mathrm{r}}=19.8 \mathrm{~min}$ $\left.(3 S 4 S), t_{\mathrm{r}}=38.6 \mathrm{~min}(3 R 4 R)\right) \cdot{ }^{1} \mathrm{H} \mathrm{NMR}\left(600 \mathrm{MHz}, \mathrm{CDCl}_{3}\right) \delta 7.97$ $(\mathrm{d}, J=8.7 \mathrm{~Hz}, 2 \mathrm{H}), 7.44(\mathrm{~d}, J=8.7 \mathrm{~Hz}, 2 \mathrm{H}), 7.11-7.03(\mathrm{~m}, 10 \mathrm{H})$, $5.50(\mathrm{~d}, J=6.2 \mathrm{~Hz}, 1 \mathrm{H}), 5.05(\mathrm{~d}, J=6.3 \mathrm{~Hz}, 1 \mathrm{H}), 4.35-4.31(\mathrm{~m}$, $2 \mathrm{H}), 1.35$ (t, $J=7.1 \mathrm{~Hz}, 3 \mathrm{H}) .{ }^{13} \mathrm{C}$ NMR $\left(150 \mathrm{MHz}, \mathrm{CDCl}_{3}\right) \delta 166.0$, $165.9,141.2$, 133.8, 131.7, 130.9, 128.8, 128.3, 128.2, 128.1, 127.3, 127.1, 125.9, 116.7, 60.9, 60.6, 60.5, 14.3.

(3S,4S)-1-(4-Carboethoxyphenyl)-3-(4-methylphenyl)-4-phenyl-2azetidinone (3b). White solid, $71.7 \mathrm{mg}$, 93\% yield (cis), 88\% ee. $[\alpha]_{\mathrm{D}}^{20}=-12.1\left(c 0.72, \mathrm{CHCl}_{3}\right)$. The ee was determined by HPLC analysis using a CHIRALPAK IA column, hexane/2-propanol 90/10, flow rate $=1.0 \mathrm{~mL} \mathrm{~min}^{-1}, t_{\mathrm{r}}=13.6 \mathrm{~min}$ (major) and $t_{\mathrm{r}}=17.7 \mathrm{~min}$ (minor). ${ }^{1} \mathrm{H}$ NMR (600 MHz, $\left.\mathrm{CDCl}_{3}\right) \delta 7.97$ (d, $\left.J=8.5 \mathrm{~Hz}, 2 \mathrm{H}\right), 7.43$ $(\mathrm{d}, J=8.5 \mathrm{~Hz}, 2 \mathrm{H}), 7.13-7.11(\mathrm{~m}, 3 \mathrm{H}), 7.05-7.04(\mathrm{~m}, 2 \mathrm{H}), 6.93-6.88$ $(\mathrm{m}, 4 \mathrm{H}), 5.48(\mathrm{~d}, J=6.2 \mathrm{~Hz}, 1 \mathrm{H}), 5.02(\mathrm{~d}, J=6.2 \mathrm{~Hz}, 1 \mathrm{H}), 4.35-4.32$ $(\mathrm{m}, 2 \mathrm{H}), 2.17(\mathrm{~s}, 3 \mathrm{H}), 1.36(\mathrm{t}, J=7.1 \mathrm{~Hz}, 3 \mathrm{H}) .{ }^{13} \mathrm{C} \mathrm{NMR}(150 \mathrm{MHz}$, $\left.\mathrm{CDCl}_{3}\right) \delta 166.3,166.0,141.2,137.0,133.9,130.9,128.9,128.7,128.5$, 128.4, 128.1, 127.1, 125.8, 116.7, 60.9, 60.6, 60.4, 21.1, 14.3.

(3S,4S)-1-(4-Carboethoxyphenyl)-3-(3-methylphenyl)-4-phenyl-2azetidinone (3c). White solid, $70.2 \mathrm{mg}, 91 \%$ yield (cis), 91\% ee. $[\alpha]_{\mathrm{D}}^{20}=-11.4\left(c 0.69, \mathrm{CHCl}_{3}\right)$. The ee was determined by HPLC analysis using a CHIRALPAK IA column, hexane/2-propanol 90/10,

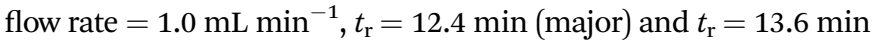
(minor). ${ }^{1} \mathrm{H}$ NMR (600 MHz, $\left.\mathrm{CDCl}_{3}\right) \delta 7.97$ (d, $\left.J=8.5 \mathrm{~Hz}, 2 \mathrm{H}\right), 7.44$ $(\mathrm{d}, J=8.5 \mathrm{~Hz}, 2 \mathrm{H}), 7.12-6.95(\mathrm{~m}, 6 \mathrm{H}), 6.86-6.80(\mathrm{~m}, 3 \mathrm{H}), 5.49(\mathrm{~d}$, $J=6.2 \mathrm{~Hz}, 1 \mathrm{H}), 5.02$ (d, $J=6.2 \mathrm{~Hz}, 1 \mathrm{H}), 4.36-4.32(\mathrm{~m}, 2 \mathrm{H}), 2.16$ (s, $3 \mathrm{H}), 1.36$ (t, $J=7.1 \mathrm{~Hz}, 3 \mathrm{H}) .{ }^{13} \mathrm{C} \mathrm{NMR}\left(150 \mathrm{MHz}, \mathrm{CDCl}_{3}\right) \delta 166.1$, 166.0, 141.2, 137.7, 133.9, 131.5, 130.9, 129.5, 128.3, 128.1, 128.03, 128.00, 127.1, 125.9, 125.8, 116.7, 60.9, 60.6, 60.6, 21.2, 14.3.

(3S,4S)-1-(4-Carboethoxyphenyl)-3-(4-fluorophenyl)-4-phenyl-2azetidinone (3d). White solid, $57.6 \mathrm{mg}, 74 \%$ yield (cis), $88 \%$ ee. $[\alpha]_{\mathrm{D}}^{20}=-5.6\left(c 0.3, \mathrm{CHCl}_{3}\right)$. The ee was determined by HPLC analysis using a CHIRALPAK IA column, hexane/2-propanol 85/15, flow rate $=1.0 \mathrm{~mL} \mathrm{min^{-1 }}, t_{\mathrm{r}}=16.7 \mathrm{~min}$ (major) and $t_{\mathrm{r}}=14.3 \mathrm{~min}$ (minor). ${ }^{1} \mathrm{H}$ NMR (600 MHz, $\left.\mathrm{CDCl}_{3}\right) \delta 7.98(\mathrm{~d}, J=8.7 \mathrm{~Hz}, 2 \mathrm{H}), 7.43$ (d, $J=8.7 \mathrm{~Hz}, 2 \mathrm{H}), 7.14-7.13(\mathrm{~m}, 3 \mathrm{H}), 7.03-7.01(\mathrm{~m}, 4 \mathrm{H}), 6.80-6.77$ $(\mathrm{m}, 2 \mathrm{H}), 5.50(\mathrm{~d}, J=6.2 \mathrm{~Hz}, 2 \mathrm{H}), 5.03(\mathrm{~d}, J=6.2 \mathrm{~Hz}, 1 \mathrm{H}), 4.36-4.32$ $(\mathrm{m}, 2 \mathrm{H}), 1.36$ (t, $J=7.1 \mathrm{~Hz}, 3 \mathrm{H}) .{ }^{13} \mathrm{C} \mathrm{NMR}$ (150 MHz, $\mathrm{CDCl}_{3}$ ) $\delta$ 166.0, 165.7, 162.8, 161.1, 141.1, 133.7, 130.9, 130.5, 130.4, $128.51,128.3,127.0,126.0,116.8,115.3,115.1,60.9,60.5,59.8$, 14.3 .

(3S,4S)-1-(4-Carboethoxyphenyl)-3-(cyclohexyl)-4-phenyl-2azetidinone (3e). White solid, $51 \mathrm{mg}$, 68\% yield (cis), 12\% ee. $[\alpha]_{\mathrm{D}}^{20}=-11.8\left(c 0.57, \mathrm{CHCl}_{3}\right)$. The ee was determined by HPLC analysis using a CHIRALPAK IA column, hexane/2-propanol 95/

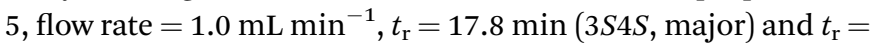
$15.6 \mathrm{~min}(3 R 4 R$, minor) (ref. 12: CHIRALPAK IA column, hexane/

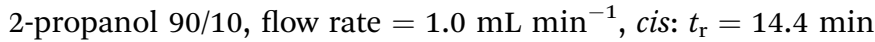
$(3 R 4 R), t_{\mathrm{r}}=16.6 \mathrm{~min}(3 S 4 S)$; trans: $\left.t_{\mathrm{r}}=19.6 \mathrm{~min}, t_{\mathrm{r}}=25.9 \mathrm{~min}\right)$. ${ }^{1} \mathrm{H} \mathrm{NMR}\left(600 \mathrm{MHz}, \mathrm{CDCl}_{3}\right) \delta 7.91(\mathrm{~d}, J=8.1 \mathrm{~Hz}, 2 \mathrm{H}), 7.44-7.28$ $(\mathrm{m}, 7 \mathrm{H}), 5.18(\mathrm{~d}, J=5.9 \mathrm{~Hz}, 1 \mathrm{H}), 4.31(\mathrm{~d}, J=7.0 \mathrm{~Hz}, 2 \mathrm{H}), 3.37-$ $3.34(\mathrm{~m}, 1 \mathrm{H}), 2.21(\mathrm{~s}, 1 \mathrm{H}), 1.66(\mathrm{~s}, 2 \mathrm{H}), 1.51(\mathrm{~s}, 2 \mathrm{H}), 1.34(\mathrm{t}, J=$
$7.2 \mathrm{~Hz}, 3 \mathrm{H}), 1.18-1.10(\mathrm{~m}, 4 \mathrm{H}), 0.76-0.66$ (m, 2H). ${ }^{13} \mathrm{C}$ NMR (150 $\left.\mathrm{MHz}, \mathrm{CDCl}_{3}\right) \delta 167.8,166.1,141.1,137.2,134.6,130.8,129.0$, 128.7, 128.6, 125.3, 124.9, 116.6, 115.9, 62.9, 60.8, 60.7, 60.7, 58.6, 34.9, 31.1, 30.7, 29.7, 29.7, 26.1, 25.5, 25.4, 14.3.

(3S,4S)-1-(4-Carboethoxyphenyl)-4-(3-methylphenyl)-3-phenyl-2azetidinone (3f). White solid, $61.7 \mathrm{mg}, 80 \%$ yield (cis), 90\% ee. $[\alpha]_{\mathrm{D}}^{20}=-11.5\left(c 0.93, \mathrm{CHCl}_{3}\right)$. The ee was determined by HPLC analysis using a Chiralcel OD-H column, hexane/2-propanol 90/10, flow rate $=0.8 \mathrm{~mL} \mathrm{~min}^{-1}, t_{\mathrm{r}}=15.4 \mathrm{~min}$ (major) and $t_{\mathrm{r}}=20.0 \mathrm{~min}$ (minor). ${ }^{1} \mathrm{H}$ NMR (600 MHz, $\mathrm{CDCl}_{3}$ ) $\delta 7.97$ (d, $\left.J=8.5 \mathrm{~Hz}, 2 \mathrm{H}\right), 7.44$ $(\mathrm{d}, J=8.5 \mathrm{~Hz}, 2 \mathrm{H}), 7.10-7.04(\mathrm{~m}, 5 \mathrm{H}), 6.98(\mathrm{t}, J=7.8 \mathrm{~Hz}, 1 \mathrm{H}), 6.89$ $(\mathrm{d}, J=7.6 \mathrm{~Hz}, 1 \mathrm{H}), 6.83(\mathrm{~d}, J=6.3 \mathrm{~Hz}, 2 \mathrm{H}), 5.45(\mathrm{~d}, J=6.2 \mathrm{~Hz}, 1 \mathrm{H})$, $5.03(\mathrm{~d}, J=6.2 \mathrm{~Hz}, 1 \mathrm{H}), 4.33(\mathrm{q}, J=7.1 \mathrm{~Hz}, 2 \mathrm{H}), 3.14(\mathrm{~s}, 3 \mathrm{H}), 1.35(\mathrm{t}$, $J=7.1 \mathrm{~Hz}, 3 \mathrm{H}) .{ }^{13} \mathrm{C} \mathrm{NMR}\left(150 \mathrm{MHz}, \mathrm{CDCl}_{3}\right) \delta 166.04,166.00,141.3$, 138.0, 134.0, 131.8, 130.8, 128.9, 128.8, 128.2, 128.1, 127.7, 125.8, 124.2, 116.7, 60.9, 60.6, 60.5, 21.2, 14.3. ESI-HRMS calcd for $\mathrm{C}_{25} \mathrm{H}_{24} \mathrm{NO}_{3}{ }^{+}[\mathrm{M}+\mathrm{H}]^{+}:$386.1751, found 386.1725.

(3S,4S)-1-(4-Carboethoxyphenyl)-4-(4-methylphenyl)-3-phenyl-2azetidinone (3g). White solid, $62.4 \mathrm{mg}, 81 \%$ yield (cis), $83 \%$ ee. $[\alpha]_{\mathrm{D}}^{20}=-18.3\left(c 0.9, \mathrm{CHCl}_{3}\right)$. The ee was determined by HPLC analysis using a Chiralcel OD-H column, hexane/2-propanol 90/10,

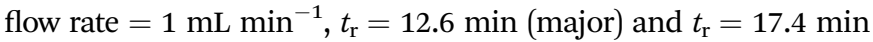
(minor). ${ }^{1} \mathrm{H}$ NMR (600 MHz, $\left.\mathrm{CDCl}_{3}\right) \delta 7.96(\mathrm{~d}, J=8.8 \mathrm{~Hz}, 2 \mathrm{H}), 7.43$ $(\mathrm{d}, J=8.6 \mathrm{~Hz}, 2 \mathrm{H}), 7.11-7.05(\mathrm{~m}, 5 \mathrm{H}), 6.93-6.90$ (m, 4H), 5.46 (d, $J$ $=6.2 \mathrm{~Hz}, 1 \mathrm{H}), 5.03(\mathrm{~d}, J=6.2 \mathrm{~Hz}, 1 \mathrm{H}), 4.35-4.31(\mathrm{~m}, 2 \mathrm{H}), 2.18(\mathrm{~s}$, $3 \mathrm{H}), 1.35(\mathrm{t}, J=7.1 \mathrm{~Hz}, 3 \mathrm{H}) .{ }^{13} \mathrm{C} \mathrm{NMR}\left(150 \mathrm{MHz}, \mathrm{CDCl}_{3}\right) \delta 166.1$, 166.0, 141.2, 137.9, 131.9, 130.8, 130.7, 129.1, 128.8, 128.2, 127.3, 127.0, 125.8, 116.8, 60.8, 60.5, 60.5, 21.1, 14.3.

(3S,4S)-1-(4-Carboethoxyphenyl)-4-(2-naphthyl)-3-phenyl-2azetidinone (3h). Light yellow solid, $59 \mathrm{mg}$, 70\% yield (cis), 77\% ee. $[\alpha]_{\mathrm{D}}^{20}=-25.5\left(c 0.53, \mathrm{CHCl}_{3}\right)$. The ee was determined by HPLC analysis using a CHIRALPAK IA column, hexane/2-propanol 90/10,

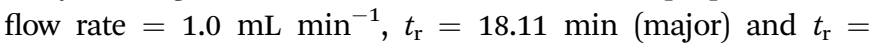
$21.51 \mathrm{~min}$ (minor). ${ }^{1} \mathrm{H} \mathrm{NMR}\left(600 \mathrm{MHz}, \mathrm{CDCl}_{3}\right) \delta 7.95(\mathrm{~d}, J=8.8 \mathrm{~Hz}$, 2H), 7.69-7.62 (m, 3H), 7.54 (d, $J=8.7 \mathrm{~Hz}, 1 \mathrm{H}), 7.47$ (d, $J=8.8 \mathrm{~Hz}$, $2 \mathrm{H}), 7.43-7.38(\mathrm{~m}, 2 \mathrm{H}), 7.10-6.95(\mathrm{~m}, 6 \mathrm{H}), 5.65(\mathrm{~d}, J=6.2 \mathrm{~Hz}, 1 \mathrm{H})$, $5.13(\mathrm{~d}, J=6.2 \mathrm{~Hz}, 1 \mathrm{H}), 4.34-4.30(\mathrm{~m}, 2 \mathrm{H}), 1.34(\mathrm{t}, J=7.1 \mathrm{~Hz}, 3 \mathrm{H})$. ${ }^{13} \mathrm{C}$ NMR $\left(150 \mathrm{MHz}, \mathrm{CDCl}_{3}\right) \delta 166.04,165.96,141.3,132.9,132.9$, 131.6, 131.6, 130.9, 128.7, 128.2, 128.2, 127.8, 127.7, 127.4, 126.7, 126.3, 126.3, 125.9, 124.2, 116.7, 60.9, 60.8, 60.7, 14.3.

(3S,4S)-1-(4-Carboethoxyphenyl)-3-(4-methylphenyl)-4-(3methylphenyl)-2-azetidinone (3i). White solid, $72.7 \mathrm{mg}, 91 \%$ yield (cis), $89 \%$ ee. $[\alpha]_{\mathrm{D}}^{20}=-16.0\left(c 1.09, \mathrm{CHCl}_{3}\right)$. The ee was determined by HPLC analysis using a Chiralcel OD-H column, hexane/2-propanol 90/10, flow rate $=1 \mathrm{~mL} \mathrm{~min}^{-1}, t_{\mathrm{r}}=$ $11.86 \mathrm{~min}$ (major) and $t_{\mathrm{r}}=14.50 \mathrm{~min}$ (minor). ${ }^{1} \mathrm{H}$ NMR (600 $\left.\mathrm{MHz}, \mathrm{CDCl}_{3}\right) \delta 7.96(\mathrm{~d}, J=8.7 \mathrm{~Hz}, 2 \mathrm{H}), 7.43(\mathrm{~d}, J=8.7 \mathrm{~Hz}, 2 \mathrm{H})$, $6.99(\mathrm{t}, J=7.9 \mathrm{~Hz}, 1 \mathrm{H}), 6.93-6.88(\mathrm{~m}, 5 \mathrm{H}), 6.84(\mathrm{~d}, J=7.2 \mathrm{~Hz}$, $2 \mathrm{H}), 5.42(\mathrm{~d}, J=6.2 \mathrm{~Hz}, 1 \mathrm{H}), 4.99(\mathrm{~d}, J=6.2 \mathrm{~Hz}, 1 \mathrm{H}), 4.35-4.31$ $(\mathrm{m}, 2 \mathrm{H}), 2.17-2.15(\mathrm{~d}, J=9.0 \mathrm{~Hz}, 6 \mathrm{H}), 1.35(\mathrm{t}, J=7.1 \mathrm{~Hz}, 3 \mathrm{H}) .{ }^{13} \mathrm{C}$ NMR (150 MHz, $\left.\mathrm{CDCl}_{3}\right) \delta 166.4,166.0,141.3,137.9,136.9,133.8$, 130.8, 128.8, 128.6, 128.2, 127.8, 125.7, 124.2, 116.7, 60.8, 60.6, 60.4, 21.3, 21.0, 14.3. ESI-HRMS calcd for $\mathrm{C}_{26} \mathrm{H}_{26} \mathrm{NO}_{3}{ }^{+}[\mathrm{M}+\mathrm{H}]^{+}$: 400.1907 , found 400.1899 .

(3S,4S)-1-(4-Carboethoxyphenyl)-3,4-(3-methylphenyl)-2azetidinone (3j). Light yellow liquid, $71.9 \mathrm{mg}$, 90\% yield (cis), $89 \%$ ee. $[\alpha]_{\mathrm{D}}^{20}=-16.9\left(c 1.32, \mathrm{CHCl}_{3}\right)$. The ee was determined by 
HPLC analysis using a Chiralcel OD-H column, hexane/2propanol 90/10, flow rate $=1 \mathrm{~mL} \min ^{-1}, t_{\mathrm{r}}=12.24 \mathrm{~min}$ (major) and $t_{\mathrm{r}}=15.45 \mathrm{~min}$ (minor). ${ }^{1} \mathrm{H} \mathrm{NMR} \mathrm{(600} \mathrm{MHz}, \mathrm{CDCl}_{3}$ ) $\delta 7.97(\mathrm{~d}, J=8.8 \mathrm{~Hz}, 2 \mathrm{H}), 7.44(\mathrm{~d}, J=8.8 \mathrm{~Hz}, 2 \mathrm{H}), 7.00-6.95(\mathrm{~m}$, $2 \mathrm{H}), 6.90-6.80(\mathrm{~m}, 6 \mathrm{H}), 5.43(\mathrm{~d}, J=6.2 \mathrm{~Hz}, 1 \mathrm{H}), 4.99(\mathrm{~d}, J=$ $6.2 \mathrm{~Hz}, 1 \mathrm{H}), 4.35-4.31(\mathrm{~m}, 2 \mathrm{H}), 2.15(\mathrm{~d}, J=5.0 \mathrm{~Hz}, 6 \mathrm{H}), 1.35(\mathrm{t}, J$ $=7.1 \mathrm{~Hz}, 3 \mathrm{H}) .{ }^{13} \mathrm{C} \mathrm{NMR}\left(150 \mathrm{MHz}, \mathrm{CDCl}_{3}\right) \delta 166.2,166.0,147.3$, $137.9,137.6,133.8,131.6,130.8,129.5,128.8,128.1,128.0$, 127.9, 127.7, 125.9, 125.7, 124.2, 116.7, 60.8, 60.6, 21.2, 21.2, 14.3. ESI-HRMS calcd for $\mathrm{C}_{26} \mathrm{H}_{26} \mathrm{NO}_{3}{ }^{+}[\mathrm{M}+\mathrm{H}]^{+}$: 400.1907, found 400.1898 .

(3S,4S)-1-(4-Carboethoxyphenyl)-3,4-(4-methylphenyl)-2azetidinone (3k). Light yellow liquid, $63.9 \mathrm{mg}$, 80\% yield (cis), $79 \%$ ee. $[\alpha]_{\mathrm{D}}^{20}=-27.2\left(c 0.94, \mathrm{CHCl}_{3}\right)$. The ee was determined by HPLC analysis using a Chiralcel OD-H column, hexane/2propanol 90/10, flow rate $=1 \mathrm{~mL} \min ^{-1}, t_{\mathrm{r}}=8.84 \mathrm{~min}$ (major) and $t_{\mathrm{r}}=11.77 \mathrm{~min}$ (minor). ${ }^{1} \mathrm{H} \mathrm{NMR}$ (600 $\mathrm{MHz}, \mathrm{CDCl}_{3}$ ) $\delta 7.95(\mathrm{~d}, J=8.8 \mathrm{~Hz}, 2 \mathrm{H}), 7.42(\mathrm{~d}, J=8.7 \mathrm{~Hz}, 2 \mathrm{H}), 6.94-6.89(\mathrm{~m}$, $8 \mathrm{H}), 5.44(\mathrm{~d}, J=6.2 \mathrm{~Hz}, 1 \mathrm{H}), 4.98(\mathrm{~d}, J=6.2 \mathrm{~Hz}, 1 \mathrm{H}), 4.35-4.31$ $(\mathrm{m}, 2 \mathrm{H}), 2.18(\mathrm{~d}, J=7.2 \mathrm{~Hz}, 6 \mathrm{H}), 1.35(\mathrm{t}, J=7.1 \mathrm{~Hz}, 3 \mathrm{H}) .{ }^{13} \mathrm{C} \mathrm{NMR}$ $\left(150 \mathrm{MHz}, \mathrm{CDCl}_{3}\right) \delta 166.4,166.0,141.3,137.8,136.8,130.8$, 129.1, 128.9, 128.7, 128.7, 127.1, 125.7, 116.7, 60.8, 60.5, 60.3, 21.1, 21.1, 14.3. ESI-HRMS calcd for $\mathrm{C}_{26} \mathrm{H}_{26} \mathrm{NO}_{3}{ }^{+}[\mathrm{M}+\mathrm{H}]^{+}$: 400.1907, found 400.1898.

(3S,4S)-1-(4-Carboethoxyphenyl)-3-(3-methylphenyl)-4-(4methylphenyl)-2-azetidinone (3l). Light yellow liquid, $65.5 \mathrm{mg}$, $82 \%$ yield (cis), $79 \%$ ee. $[\alpha]_{\mathrm{D}}^{20}=-28.1\left(c 0.89, \mathrm{CHCl}_{3}\right)$. The ee was determined by HPLC analysis using a Chiralcel OD-H column, hexane/2-propanol 90/10, flow rate $=1 \mathrm{~mL} \mathrm{~min}^{-1}, t_{\mathrm{r}}=$ $10.94 \mathrm{~min}$ (major) and $t_{\mathrm{r}}=14.99 \mathrm{~min}$ (minor). ${ }^{1} \mathrm{H}$ NMR (600 $\left.\mathrm{MHz} \mathrm{CDCl}_{3}\right) \delta 7.96(\mathrm{~d}, J=8.7 \mathrm{~Hz}, 2 \mathrm{H}), 7.43(\mathrm{~d}, J=8.7 \mathrm{~Hz}, 2 \mathrm{H})$, 6.98-6.81 (m, 8H), $5.45(\mathrm{~d}, J=6.2 \mathrm{~Hz}, 1 \mathrm{H}), 4.98(\mathrm{~d}, J=6.2 \mathrm{~Hz}$, $1 \mathrm{H}), 4.35-4.31(\mathrm{~m}, 2 \mathrm{H}), 2.17(\mathrm{~d}, J=12.0 \mathrm{~Hz}, 6 \mathrm{H}), 1.35(\mathrm{t}, J=$ $7.1 \mathrm{~Hz}, 3 \mathrm{H}) .{ }^{13} \mathrm{C} \mathrm{NMR}\left(150 \mathrm{MHz}, \mathrm{CDCl}_{3}\right) \delta 166.3,166.0,141.3$, $137.8,137.7,131.7,130.8,130.8,129.5,129.0,127.99,127.97$, 127.0, 125.9, 125.7, 116.7, 60.8, 60.50, 60.47, 21.2, 21.1, 14.3. ESI-HRMS calcd for $\mathrm{C}_{26} \mathrm{H}_{26} \mathrm{NO}_{3}{ }^{+}[\mathrm{M}+\mathrm{H}]^{+}$: 400.1907, found 400.1898 .

$(3 S, 4 S)-1,3,4$-Triphenyl-2-azetidinone $(3 \mathrm{~m})$. White solid, $56.0 \mathrm{mg}, 93 \%$ yield (cis), $91 \%$ ee. $[\alpha]_{\mathrm{D}}^{20}=-9.2\left(c 0.54, \mathrm{CHCl}_{3}\right)$. The ee was determined by HPLC analysis using a CHIRALPAK IA column, hexane/2-propanol 90/10, flow rate $=1.0 \mathrm{~mL} \mathrm{~min}^{-1}, t_{\mathrm{r}}$ $=10.35 \mathrm{~min}\left(3 S 4 S\right.$, major) and $t_{\mathrm{r}}=16.92 \mathrm{~min}(3 R 4 R$, minor). (ref. 12: CHIRALPAK IA column, hexane/2-propanol 90/10, flow

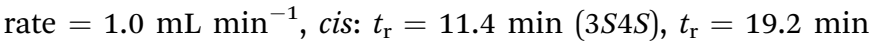
$(3 R 4 R)$; trans: $\left.t_{\mathrm{r}}=10.0 \mathrm{~min}, t_{\mathrm{r}}=13.2 \mathrm{~min}\right){ }^{1} \mathrm{H} \mathrm{NMR}(600 \mathrm{MHz}$, $\left.\mathrm{CDCl}_{3}\right) \delta$ 7.42-7.41 (m, 2H), 7.30-7.25 (m, 2H), 7.11-7.04 (m, $11 \mathrm{H}), 5.47(\mathrm{~d}, J=6.2 \mathrm{~Hz}, 1 \mathrm{H}), 5.01(\mathrm{~d}, J=6.1 \mathrm{~Hz}, 1 \mathrm{H}) .{ }^{13} \mathrm{C} \mathrm{NMR}$ $\left(150 \mathrm{MHz}, \mathrm{CDCl}_{3}\right) \delta 165.7,137.7,134.4,132.1,129.1,128.9$, 128.2, 128.1, 127.9, 127.2, 127.1, 124.1, 117.2, 60.3, 60.3.

(3S,4S)-1-(4-Methylphenyl)-3,4-diphenyl-2-azetidinone (3n). White solid, $44.5 \mathrm{mg}, 71 \%$ yield $(c i s), 85 \%$ ee. $[\alpha]_{\mathrm{D}}^{20}=-12.19(c$ $\left.0.38, \mathrm{CHCl}_{3}\right)$. The ee was determined by HPLC analysis using a CHIRALPAK IA column, hexane/2-propanol 90/10, flow rate $=$

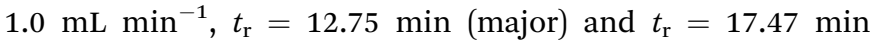
(minor). ${ }^{1} \mathrm{H}$ NMR (600 MHz, $\left.\mathrm{CDCl}_{3}\right) \delta 7.3(\mathrm{~d}, J=8.4 \mathrm{~Hz}, 2 \mathrm{H})$, 7.10-7.03 (m, 12H), 5.43 (d, $J=6.1 \mathrm{~Hz}, 1 \mathrm{H}), 4.99$ (d, $J=6.1 \mathrm{~Hz}$,
1H), 2.29 (s, 3H). ${ }^{13} \mathrm{C}$ NMR (150 MHz, $\left.\mathrm{CDCl}_{3}\right) \delta$ 165.4, 135.3, 134.5, 133.7, 132.2, 129.6, 128.9, 128.2, 128.1, 127.8, 127.13, $127.11,117.2,60.3,60.2,20.9$.

(3S,4S)-1-(4-Chlorophenyl)-3,4-diphenyl-2-azetidinone (3o). White solid, $53.4 \mathrm{mg}, 80 \%$ yield (cis), $87 \%$ ee. $[\alpha]_{\mathrm{D}}^{20}=-8.13(c$ $0.57, \mathrm{CHCl}_{3}$ ). The ee was determined by HPLC analysis using a CHIRALPAK IA column, hexane/2-propanol 90/10, flow rate = $1.0 \mathrm{~mL} \min ^{-1}, t_{\mathrm{r}}=10.66 \mathrm{~min}$ (major) and $t_{\mathrm{r}}=14.34 \mathrm{~min}$ (minor). ${ }^{1} \mathrm{H}$ NMR (600 MHz, $\left.\mathrm{CDCl}_{3}\right) \delta 7.35(\mathrm{~d}, J=8.8 \mathrm{~Hz}, 2 \mathrm{H})$, $7.23(\mathrm{~d}, J=8.8 \mathrm{~Hz}, 2 \mathrm{H}), 7.11-7.03(\mathrm{~m}, 10 \mathrm{H}), 5.44(\mathrm{~d}, J=6.1 \mathrm{~Hz}$, $1 \mathrm{H}), 5.02(\mathrm{~d}, J=6.1 \mathrm{~Hz}, 1 \mathrm{H}) .{ }^{13} \mathrm{C} \mathrm{NMR}\left(150 \mathrm{MHz}, \mathrm{CDCl}_{3}\right) \delta 165.5$, 136.2 , 133.9, 131.8, 129.2, 129.1, 128.8, 128.3, 128.1, 128.0, $127.3,127.1,118.4,60.6,60.4$.

(3S,4S)-1-(4-Bromophenyl)-3,4-diphenyl-2-azetidinone (3p). White solid, $62.0 \mathrm{mg}, 82 \%$ yield (cis), $87 \%$ ee. $[\alpha]_{\mathrm{D}}^{20}=-10.23(c$ $\left.0.72, \mathrm{CHCl}_{3}\right)$. The ee was determined by HPLC analysis using a CHIRALPAK IA column, hexane/2-propanol 90/10, flow rate = $1.0 \mathrm{~mL} \mathrm{~min}{ }^{-1}, t_{\mathrm{r}}=11.44 \mathrm{~min}$ (major) and $t_{\mathrm{r}}=15.40 \mathrm{~min}$ (minor). ${ }^{1} \mathrm{H}$ NMR (600 MHz, $\left.\mathrm{CDCl}_{3}\right) \delta 7.38(\mathrm{~d}, J=8.8 \mathrm{~Hz}, 2 \mathrm{H})$, $7.29(\mathrm{~d}, J=8.8 \mathrm{~Hz}, 2 \mathrm{H}), 7.11-7.02(\mathrm{~m}, 10 \mathrm{H}), 5.43(\mathrm{~d}, J=6.1 \mathrm{~Hz}$, $1 \mathrm{H}), 5.02(\mathrm{~d}, J=6.1 \mathrm{~Hz}, 1 \mathrm{H}) .{ }^{13} \mathrm{C} \mathrm{NMR}\left(150 \mathrm{MHz}, \mathrm{CDCl}_{3}\right) \delta 165.6$, 136.6, 133.9, 132.1, 131.8, 128.8, 128.3, 128.12, 128.06, 127.3, $127.1,118.8,116.7,60.6,60.4$.

(3S,4S)-1-(4-Fluorophenyl)-3,4-diphenyl-2-azetidinone (3q). White solid, $44.4 \mathrm{mg}, 70 \%$ yield (cis), $86 \%$ ee. $[\alpha]_{\mathrm{D}}^{20}=-7.0(c$ $0.34, \mathrm{CHCl}_{3}$ ). The ee was determined by HPLC analysis using a CHIRALPAK IA column, hexane/2-propanol 90/10, flow rate $=$ $1.0 \mathrm{~mL} \min ^{-1}, t_{\mathrm{r}}=10.43 \mathrm{~min}$ (major) and $t_{\mathrm{r}}=13.67 \mathrm{~min}$ (minor). ${ }^{1} \mathrm{H}$ NMR (600 MHz, $\mathrm{CDCl}_{3}$ ) $\delta$ 7.40-7.37 (m, 2H), 7.11$6.96(\mathrm{~m}, 12 \mathrm{H}), 5.44(\mathrm{~d}, J=6.1 \mathrm{~Hz}, 1 \mathrm{H}), 5.02(\mathrm{~d}, J=6.1 \mathrm{~Hz}, 1 \mathrm{H})$. ${ }^{13} \mathrm{C} \mathrm{NMR}\left(150 \mathrm{MHz}, \mathrm{CDCl}_{3}\right) \delta 165.3,159.9,158.3,134.1,133.9(\mathrm{~d}$, $J=10.7)$, 132.0, 128.9, 128.3, 128.1, 128.0, 127.2, 127.1, 118.66, 118.61, 116.0, 115.8, 60.53, 60.51. ESI-HRMS calcd for $\mathrm{C}_{21} \mathrm{H}_{17} \mathrm{FNO}^{+}[\mathrm{M}+\mathrm{H}]^{+}: 318.1289$, found 318.1284.

(3S,4S)-3-(4-Fluorophenyl)-1,4-diphenyl-2-azetidinone (3r). White solid, $47.6 \mathrm{mg}, 75 \%$ yield (cis), $84 \%$ ee. $[\alpha]_{\mathrm{D}}^{20}=-5.4(c$ $0.24, \mathrm{CHCl}_{3}$ ). The ee was determined by HPLC analysis using a CHIRALPAK IA column, hexane/2-propanol 85/15, flow rate $=$ $1.0 \mathrm{~mL} \mathrm{~min}^{-1}, t_{\mathrm{r}}=8.42 \mathrm{~min}$ (major) and $t_{\mathrm{r}}=15.66 \mathrm{~min}$ (minor). ${ }^{1} \mathrm{H}$ NMR $\left(600 \mathrm{MHz} \mathrm{CDCl}_{3}\right) \delta 7.41-7.40(\mathrm{~m}, 2 \mathrm{H}), 7.30-7.25(\mathrm{~m}$, 2H), 7.14-7.01 (m, 8H), 6.79-6.76 (m, 2H), 5.45 (d, $J=6.1 \mathrm{~Hz}$, $1 \mathrm{H}), 4.97(\mathrm{~d}, J=6.1 \mathrm{~Hz}, 1 \mathrm{H}) .{ }^{13} \mathrm{C} \mathrm{NMR}\left(150 \mathrm{MHz}, \mathrm{CDCl}_{3}\right) \delta 165.4$, $162.7,161.0,137.6,134.2,130.5$ (d, $J=32.5)$, 129.1, 128.4, 128.1, 127.0, 124.2, 117.2, 115.1, 115.0, 60.2, 59.5. ESI-HRMS calcd for $\mathrm{C}_{21} \mathrm{H}_{17} \mathrm{FNO}^{+}[\mathrm{M}+\mathrm{H}]^{+}:$318.1289, found 318.1290.

\section{Conflicts of interest}

There are no conflicts to declare.

\section{Acknowledgements}

We thank the National Natural Science Foundation of China (21672031), Chongqing Natural Science Foundation (cstc2019jcyjmsxmX0034 and cstc2017jcyjAX0352), and Scientific and Technological Research Program of Chongqing Municipal Education Commission (KJQN201900437) for financial support. 


\section{Notes and references}

1 (a) G. I. Georg and V. T. Ravikumar, in The Organic Chemistry of $\beta$-lactams, ed. G. I.Georg, VCH Publishers, New York, 1992, pp. 295-368; (b) Heterocyclic Scaffolds I: $\beta$-Lactams, ed. B. K.Bank, Springer, 2010; (c) $\beta$-Lactams: Unique Structures of Distinction for Novel Molecules, ed. B. K.Bank, Springer, 2013; (d) J. D. Fisher and S. Mobashery, The $\beta$-Lactam (Azetidin-2-one) as a privileged Ring in Medicinal Chemistry, in Privileged Scaffolds in Medical Chemistry: Design, Synthesis, Evaluation, ed. S.Bräae, The Royal Society of Chemistry, UK, 2015, ch. 3, pp. 64-97.

2 For selected examples of drugs or potent drugs containing $\beta$ lactams, see: (a) T. Kosoglou, P. Statkevich, A. O. JohnsonLevonas, J. F. Paolini, A. J. Bergman and K. B. Alton, Clin. Pharmacokinet., 2005, 44, 467-494; (b) W. Wang, P. Devasthale, D. Farrelly, L. Gu, T. Harrity, M. Cap, C. Chu, L. Kunselman, N. Morgan, R. Ponticiello, R. Zebo, L. Zhang, K. Locke, J. Lippy, K. O’ Malley, V. Hosagrahara, L. Zhang, P. Kadiyala, C. Chang, J. Muckelbauer, A. M. Doweko, R. Zahler, D. Ryono, N. Hariharan and P. T. W. Cheng, Bioorg. Med. Chem. Lett., 2008, 18, 19391944; (c) D. Xiao, A. Palani, R. Aslanian, B. Mckittrick, A. T. McPhail, C. C. Correll, P. T. Phelps, J. C. Anthes and D. Rindgen, Bioorg. Med. Chem. Lett., 2009, 19, 783-787; (d) M. Feledziaka, C. Michaux, D. M. Lambert and J. Marchand-Brynaert, Eur. J. Med. Chem., 2013, 60, 101111; (e) X. Cui, L. Li, Y. Y. Hu, S. Ren, M. Zhang and W. B. Li, Mol. Neurobiol., 2015, 51, 1322-1333.

3 B. Alcaide, P. Almendros and C. Aragoncillo, Chem. Rev., 2007, 107, 4437-4492.

4 (a) S. Hosseyni and A. Jarrahpour, Org. Biomol. Chem., 2018, 16, 6840-6852; (b) C. R. Pitts and T. Lectka, Chem. Rev., 2014, 114, 7930-7953; (c) P. A. Magriotis, Eur. J. Org. Chem., 2014, 2647-2657; (d) S. France, A. Weatherwax, A. E. Taggi and T. Lectka, Acc. Chem. Res., 2004, 37, 592-600; (e) G. S. Singh, Tetrahedron, 2003, 59, 7631-7649.

5 M. Kinugasa and S. J. Hashimoto, J. Chem. Soc., Chem. Commun., 1972, 466-467.

6 For recent reviews, see: (a) S. Murahashi and Y. Imada, Chem. Rev., 2019, 119, 4684-4716; (b) J. Comas-Barceló and J. P. A. Harrity, Synthesis, 2017, 49, 1168-1181; (c) T. Hashimoto and K. Maruoka, Chem. Rev., 2015, 115, 5366-5412; (d) M. Chigrinova, D. A. MacKenzie, A. R. Sherratt, L. L. W. Cheung and J. P. Pezacki, Molecules, 2015, 20, 6959-6969; (e) S. Stecko, B. Furman and M. Chmielewski, Tetrahedron, 2014, 70, 7817-7844; $(f)$ R. H. Khangarot and K. P. Kaliappan, Eur. J. Org. Chem., 2013, 7664-7677; (g) M. Maciejko, S. Stecko, O. StaszewskaKrajewska, M. Jurczak, B. Furman and M. Chmielewski, Synthesis, 2012, 44, 2825-2839.

7 M. Miura, M. Enna, K. Okuro and M. Nomira, J. Org. Chem., 1995, 60, 4999-5004.

8 (a) M. M. C. Lo and G. C. Fu, J. Am. Chem. Soc., 2002, 124, 4572-4573; (b) R. Shintani and G. C. Fu, Angew. Chem., Int. Ed., 2003, 42, 4082-4085.
9 (a) M. C. Ye, J. Zhou, Z. Z. Huang and Y. Tang, Chem. Commun., 2003, 2554-2555; (b) M. C. Ye, J. Zhou and Y. Tang, J. Org. Chem., 2006, 71, 3576-3582; (c) J. H. Chen, S. H. Liao, X. L. Sun, Q. Shen and Y. Tang, Tetrahedron, 2012, 68, 5042-5045.

10 A. G. Coyne, H. Müller-Bunz and P. J. Guiry, Tetrahedron: Asymmetry, 2007, 18, 199-207.

11 (a) D. A. Evans, F. Kleinbeck and M. Rüping, in Asymmetric Synthesis - The Essentials, ed. M.Christmann and S.Bräse, Wiley-VCH, Weinheim, Germany, 2007, pp. 72-77; (b) T. Saito, T. Kikichi, H. Tanabe, J. Yahiro and T. Otani, Tetrahedron Lett., 2009, 50, 4969-4972; (c) B. Baeza, L. Casarrubios and M. A. Sierra, Chem.-Eur. J., 2013, 19, 11536-11540.

12 Z. Chen, L. Lin, M. Wang, X. Liu and X. Feng, Chem.-Eur. J., 2013, 19, 7561-7567.

13 K. Wolosewicz, M. Michalak, J. Adamek and B. Furman, Eur. J. Org. Chem., 2016, 2212-2219.

14 (a) Y. Takayama, T. Ishii, H. Ohmiya, T. Iwai, M. C. Schwarzer, S. Mori, T. Taniguchi, K. Monde and M. Sawamura, Chem.-Eur. J., 2017, 23, 8400-8404; (b) K. Imai, Y. Takayama, H. Murayama, H. Ohmiya, Y. Shimizu and M. Sawamura, Org. Lett., 2019, 21, 1717-1721.

15 T. Shu, L. Zhao, S. Li, X.-Y. Chen, C. von Essen, K. Rissanen and D. Enders, Angew. Chem., Int. Ed., 2018, 57, 1098510988.

16 For recent reviews about imine-containing ligands, see: X. Chen and Z. Lu, Org. Biomol. Chem., 2017, 15, 2280-2306.

17 (a) B. Liu, J. Liu, X. Jia, L. Huang, X. Li and A. S. C. Chan, Tetrahedron: Asymmetry, 2007, 18, 1124-1128; (b) B. Liu, L. Huang, Y. Zhong, X. Li and A. S. C. Chan, Tetrahedron: Asymmetry, 2007, 18, 2901-2904; (c) B. Liu, Y. Zhong and X. Li, Chirality, 2009, 21, 595-599.

18 (a) S. Zhang, P. Deng, J. Zhou, M. Liu, G. Liang, Y. Xiong and H. Zhou, Chem. Commun., 2017, 53, 12914-12917; (b) G. Chen, G. Liang, Y. Wang, P. Deng and H. Zhou, Org. Biomol. Chem., 2018, 16, 3841-3850.

19 For reviews, see: J. Barrios-Rivera, Y. Xu and M. Wills, Org. Biomol. Chem., 2019, 17, 1301-1321.

20 For detailed data, see ESI. $\dagger$

21 The results of other solvents in the presence of $\mathrm{Cu}(\mathrm{OTf})_{2} / \mathbf{L 1}$ : $\mathrm{CH}_{2} \mathrm{Cl}_{2}$ (no reaction), THF (no reaction), toluene (no reaction).

22 The results of other solvents in the presence of CuOTf/L2: $\mathrm{CH}_{2} \mathrm{Cl}_{2}$ (44\% yield, 98/2 cis/trans, $18 \%$ cis ee), THF (70\% yield, 96/4 cis/trans, $6 \%$ cis ee), toluene (no reaction).

23 For recent reports: (a) T. C. Malig, D. Yu and J. E. Hein, J. Am. Chem. Soc., 2018, 140, 9167-9173; (b) S. Santoro, R. Liao, T. Marcelli, P. Hammar and F. Himo, J. Org. Chem., 2015, 80, 2649-2660.

24 DFT calculations were carried out using the Gaussian 09 software package. For more detailed results about the optimized structure using DFT, see ESI. $\dagger$

25 For a related mechanistic research in asymmetric Staudinger reaction, see: S. France, M. H. Shah, A. Weatherwax, H. Wack, J. P. Roth and T. Lectka, J. Am. Chem. Soc., 2005, 127, 1206-1215. 